\title{
Fast and Accurate Detection of COVID-19 Along With 14 Other Chest Pathologies Using a Multi-Level Classification: Algorithm Development and Validation Study
}

\author{
Saleh Albahli ${ }^{1,2^{*}}$, BSc, PhD; Ghulam Nabi Ahmad Hassan Yar $^{3^{*}}$, BS, MS \\ ${ }^{1}$ Department of Information Technology, College of Computer, Qassim University, Buraydah, Saudi Arabia \\ ${ }^{2}$ Department of Computer Science, Kent State University, Kent, OH, United States \\ ${ }^{3}$ Depratment of Electrical and Computer Engineering, Air University, Islamabad, Pakistan \\ *all authors contributed equally
}

Corresponding Author:

Saleh Albahli, BSc, PhD

Department of Information Technology

College of Computer

Qassim University

Buraydah, 51452

Saudi Arabia

Phone: 966163012604

Email: salbahli@qu.edu.sa

\section{Abstract}

Background: COVID-19 has spread very rapidly, and it is important to build a system that can detect it in order to help an overwhelmed health care system. Many research studies on chest diseases rely on the strengths of deep learning techniques. Although some of these studies used state-of-the-art techniques and were able to deliver promising results, these techniques are not very useful if they can detect only one type of disease without detecting the others.

Objective: The main objective of this study was to achieve a fast and more accurate diagnosis of COVID-19. This study proposes a diagnostic technique that classifies COVID-19 x-ray images from normal x-ray images and those specific to 14 other chest diseases.

Methods: In this paper, we propose a novel, multilevel pipeline, based on deep learning models, to detect COVID-19 along with other chest diseases based on x-ray images. This pipeline reduces the burden of a single network to classify a large number of classes. The deep learning models used in this study were pretrained on the ImageNet dataset, and transfer learning was used for fast training. The lungs and heart were segmented from the whole $\mathrm{x}$-ray images and passed onto the first classifier that checks whether the x-ray is normal, COVID-19 affected, or characteristic of another chest disease. If it is neither a COVID-19 x-ray image nor a normal one, then the second classifier comes into action and classifies the image as one of the other 14 diseases.

Results: We show how our model uses state-of-the-art deep neural networks to achieve classification accuracy for COVID-19 along with 14 other chest diseases and normal cases based on X-ray images, which is competitive with currently used state-of-the-art models. Due to the lack of data in some classes such as COVID-19, we applied 10-fold cross-validation through the ResNet50 model. Our classification technique thus achieved an average training accuracy of $96.04 \%$ and test accuracy of $92.52 \%$ for the first level of classification (ie, 3 classes). For the second level of classification (ie, 14 classes), our technique achieved a maximum training accuracy of $88.52 \%$ and test accuracy of $66.634 \%$ by using ResNet50. We also found that when all the 16 classes were classified at once, the overall accuracy for COVID-19 detection decreased, which in the case of ResNet50 was $88.92 \%$ for training data and $71.905 \%$ for test data.

Conclusions: Our proposed pipeline can detect COVID-19 with a higher accuracy along with detecting 14 other chest diseases based on X-ray images. This is achieved by dividing the classification task into multiple steps rather than classifying them collectively.

(J Med Internet Res 2021;23(2):e23693) doi: 10.2196/23693 


\section{KEYWORDS}

COVID-19; chest x-ray; convolutional neural network; data augmentation; biomedical imaging; automatic detection

\section{Introduction}

\section{Background}

The COVID-19 pandemic has been causing significant health concerns since 2019. Symptoms of the disease include fever, cough, headache, and severe respiratory complications, which can subsequently lead to death. When this disease first started to spread in December 2019, numerous unknown facts were reported in Wuhan, China, where the first outbreak occurred [1]. By early January 2020, the government of China and the World Health Organization recognized SARS-CoV-2, the novel coronavirus known to cause COVID-19, as a pathogenic virus that belongs to the same family (Coronaviridae) as the virus known to cause severe acute respiratory syndrome (SARS). A SARS outbreak was previously reported in China in 2002-2003 [2].

Medical x-rays (short for X-radiation) are a form of visible light rays but with higher energy that penetrate the body to generate images of tissues and structures within the body, including bones, chest, and teeth. X-ray imaging is a very effective diagnostic tool and has been used for several decades by specialists to detect fractures, certain tumors, pneumonia, and dental problems [3]. In advanced cases, computed tomography (CT) can be used to produce a series of body images, which is later assembled into a $3 \mathrm{D} x$-ray image that is processed by a computer. However, the traditional x-ray is a lot faster, easier, cheaper, and less harmful than a CT scan [4].

Research has shown that deep learning can be used to make predictions based on medical images by extracting characteristic features, including the shape and spatial rotation, from the images. Convolutional neural networks (CNNs) have played a very vital role in feature extraction and learning patterns that enable prediction. For example, a $\mathrm{CNN}$ is used to improve extraction high-speed video-endoscopy when the training data is very limited [5]. Advancements in image processing tools have brought about a radical change in the current techniques for the detection of pulmonary diseases. Researchers are employing traditional computer vision as well as deep learning algorithms to achieve satisfactory performance [3]. Several primary benefits are strongly correlated with the advancement of radiographic image classification tools. For example, in rural areas, owing to a shortage of doctors and places where doctors cannot be reached, such tools can prove useful. Once these tools become pervasive in the health care industry, radiologists, clinic practitioners, and even patients may utilize radiographic image classification tools to monitor and treat several diseases. As a result, this can reduce the burden on radiologists all over the world, by abolishing the requirement to examine every $\mathrm{x}$-ray image for anomalies. Instead, the doctors will only need to focus on the patients whose $\mathrm{x}$-ray images are flagged by this tool. The use of such tools can also eliminate the subjective opinion of doctors, increase the speed of early diagnosis of disease, and identify the minor details that may be overlooked by the human eye in some cases.
For this study, owing to computational restraints, we did not build a model from scratch, as such models require extremely high-end computers. Rather, we used CNN as a class of deep neural networks to propose a model to classify COVID-19 x-ray images from x-ray images of a wide range of chest diseases. Although the x-ray images of the other diseases are inadequate for proper training and to achieve state-of-the-art results, we generalized the data by considering data augmentation. This mainly rescales the x-ray images and flips them horizontally, in addition to a few other functionalities such as shift range, zooming, and rotation.

The strength of this study is that it classifies X-ray images at two different stages. The first stage involves enhancing the model to detect COVID-19-specific x-ray images at a faster speed than $x$-ray images of other chest diseases. This will result in a significant increase in the classification speed. Thus, considering a part of a dataset of chest $\mathrm{x}$-ray (CXR) images for the analysis will result in low-quality output and unsatisfactory diagnoses. Accordingly, if the case is not classified as "normal" or "COVID-19" at this stage, then the classification is continued to the second stage, which involves classification for 14 other chest and related conditions (ie, atelectasis, cardiomegaly, effusion, infiltration, mass, nodule, pneumonia, pneumothorax, consolidation, edema, emphysema, fibrosis, pleural, and hernia). This also saves processing power if the $\mathrm{x}$-ray image has been classified as "normal" or "COVID-19" in the first stage itself. To further enhance the accuracy of detection, we used UNet to complete lung and heart segmentation. Because we used a pretrained model, we were able to independently train 5 different models for each stage. Models with the best training and test accuracy were then selected for further analyses.

Based on our findings, we found that ResNet50 is the best model for classification in both scenarios: classifying 3 classes and 14 classes. Moreover, image segmentation helps in increasing the classification accuracy by up to $5 \%$. We also trained a model for all 16 classes and found that classifying for a large number of classes significantly reduces the overall accuracy of the model.

The main contributions of this study are as follows:

1. Introduction of new classification pipeline for more accurate, automated classification in case of a large number of classes, primarily to increase the accuracy of a specific class.

2. Use of augmentation and semantic segmentation to increase accuracy of the model.

3. Comparison between different deep learning models on the basis of classification in cases of small and large number of classes.

In this paper, we first review previous studies that used deep neural networks for the detection of COVID-19 and other chest diseases. Then, we discuss the datasets used for our experiments as well as the study methodology, including data preprocessing, data segmentation, and the setup for classification of the models. 
Finally, we present the results and analyses on the basis of the models and dataset available.

\section{Previous Work}

Recently, with the rapid development of artificial intelligence, an increasing number of researchers have begun to pay attention to intelligent, deep learning-based diagnostic techniques. Some of them have achieved significantly prominent results. In this section, we first review the current, state-of-the-art techniques concerning the application of artificial intelligence to chest diseases in general, and then, we discuss the literature related to COVID-19 detection using deep neural networks.

\section{Detection of Chest Diseases Based on CXR Images by Using Deep Neural Networks}

Sivasamy and Subashini [6] used a Keras framework to classify CXR images to predict lung diseases and reported an accuracy of $86.14 \%$. The accuracy of the model improved as the number of epochs for training was increased. Wang et al [7] used pixel-wise annotated digital reconstructed radiograph data to train an unsupervised multiorgan segmentation model based on $\mathrm{x}$-ray images. In this case, the gaps in nodules annotated directly on 2D x-ray images are quite challenging and time-consuming due to the projective nature of $\mathrm{x}$-ray imaging. Rajpurkar et al [8] proposed a binary classifier for the detection of pneumonia from frontal-view CXR images that achieved an $\mathrm{f1}$ score of 0.435. Salehinejad et al [9] used a Deep Convolutional Generative Adversarial Network (DCGAN) tailored model designed for training with $\mathrm{X}$-ray images wherein a generator is trained to generate artificial CXR images. Their model obtained its best accuracy when trained on an augmented dataset with DCGAN-synthesized CXRs to balance the imbalanced real dataset (D3). Chandra and Verma [10] used 5 different models to identify pneumonia and reported $95.631 \%$ as the best accuracy. The model is limited to analyzing only nonrigid, deformable, registration-driven automatically lung regions and segmented region of interest-confined feature extraction. Previous studies using state-of-the-art techniques have achieved effective results with one or two cardiothoracic diseases, but these techniques could lead to misclassification.

A few techniques have targeted all 14 classes of chest diseases. Wang et al [11] presented the largest publicly available dataset of CXR images, which has provided a new dimension to the research community. They achieved promising results using a deep CNN and suggest that this dataset could be further extended by using more disease labels. Smit et al [12] proposed a deep learning-based technique to identify the 14 underlying chest diseases. They trained the model to input a single-view chest radiograph and output the probability of each of the 14 observations. Several models were trained to identify the one with the best accuracy. They used DenseNet121 for their research and found that it yielded the best accuracy, but it was limited to the CheXpert dataset and liable to overfitting. A pretrained DenseNet121 model and feature extraction techniques were used for accurate identification of 14 thoracic diseases in the study by Ho and Gwak [13].

\section{Detection of COVID-19 Cases Based on CXR Images by Using Deep Neural Networks}

There are several state-of-the-art studies on deep learning and machine learning models for COVID-19 diagnosis. A study by Apostolopoulos and Mpesiana [14] took advantage of CNNs for the automatic detection of COVID-19 by using CXR images. They adopted transfer learning to solve for the small image dataset challenge. Their COVID-19 dataset consisted of 224 sample medical images. Despite the size limitation, their results showed effective automatic detection of COVID-19-related diseases. Abbas et al [15] used the CNN-based DeTraC framework. They also used transfer learning to achieve the best performance. This model achieved $95.12 \%$ accuracy and $97.91 \%$ sensitivity. Chen et al [16] provided a prediction of patients with or without COVID-19 by using the UNet++ based segmentation model. Narin et al [17] classified CXR images using the ResNet50 model and obtained the highest classification performance with $98 \%$ accuracy, using a dataset comprising only 50 COVID-19 and 50 normal samples. Li et al [18] also used a ResNet50 model with a dataset comprising 468 COVID-19 samples, 1551 community-acquired pneumonia samples, and 1445 non-pneumonia samples; this model achieved $90 \%$ sensitivity. Using deep learning approaches to extract and transform features, Li et al proved their model's efficacy in COVID-19 diagnosis [18]. Furthermore, Sethy and Behera [19] used deep learning to extract deep features from x-ray images and then used state vector machine to classify them into COVID-19-positive and COVID-19-negative classes; they achieved an accuracy of $95 \%$. Hemdan et al [20] used transfer learning and fine-tuning on state-of-the-art networks like VGG and ResNetV2 to classify COVID-19-positive and COVID-19-negative x-ray images; they achieved an accuracy of $90 \%$. Wang et al [21] proposed the M-inception model, a variant of the inception model. They detected only COVID-19 CT images from all available images and achieved an accuracy of $82 \%$. Table 1 presents a comparison of previously studies models using radiographic imaging classification for COVID-19 cases, normal cases, and other chest diseases. 
Table 1. Comparison of models detecting COVID-19 cases, normal cases, and other chest diseases based on medical images (data derived from [22]).

\begin{tabular}{|c|c|c|c|c|c|c|c|}
\hline \multirow[t]{2}{*}{ Reference } & \multirow[t]{2}{*}{ Medical image } & \multicolumn{3}{|c|}{ Disease detected, $\mathrm{n}$} & \multirow[t]{2}{*}{ Accuracy $(\%)$} & \multirow[t]{2}{*}{ Methodology } & \multirow[t]{2}{*}{ Gaps in classification } \\
\hline & & $\begin{array}{l}\text { COVID- } \\
19\end{array}$ & Normal & $\begin{array}{l}\text { Other chest dis- } \\
\text { eases }\end{array}$ & & & \\
\hline $\begin{array}{l}\text { Apostolopoulos } \\
\text { and Mpesiana } \\
{[14]}\end{array}$ & X-ray & 224 & 504 & 700 & 93 & $\begin{array}{l}\text { Used transfer learning on } \\
\text { VGG19. MobileNetV2, } \\
\text { Inception, Xception, and } \\
\text { InceptionResNetV2 }\end{array}$ & $\begin{array}{l}\text { Used only } 3 \text { classes: } \\
\text { COVID- } 19 \text {, pneumonia, } \\
\text { and other }\end{array}$ \\
\hline Wang et al [23] & X-ray & 53 & 8066 & 5526 & 92 & $\begin{array}{l}\text { Introduced COVID- } \\
\text { Net-the first open- } \\
\text { source COVID-19 detec- } \\
\text { tion system }\end{array}$ & $\begin{array}{l}\text { Used only } 3 \text { classes: } \\
\text { COVID- } 19 \text {, pneumonia, } \\
\text { and normal }\end{array}$ \\
\hline Narin et al [17] & $\mathrm{X}$-ray & 50 & 50 & $\mathrm{~N} / \mathrm{A}^{\mathrm{a}}$ & 98 & $\begin{array}{l}\text { Used } 5 \text { pretrained net- } \\
\text { works and applied } 3 \text { bina- } \\
\text { ry classifications for } 4 \\
\text { classes of chest x-rays }\end{array}$ & $\begin{array}{l}\text { Used only } 3 \text { classes: } \\
\text { normal, COVID-19, vi- } \\
\text { ral and bacterial pneu- } \\
\text { monia }\end{array}$ \\
\hline Brunese et al [22] & X-ray & 250 & 3520 & 2753 & 97 & $\begin{array}{l}\text { Defined } 2 \text { models based } \\
\text { on VGG16: one to classi- } \\
\text { fy affected x-ray images } \\
\text { from healthy ones and } \\
\text { the other to classify } \\
\text { COVID-19 from affected } \\
\text { x-ray images. Then, they } \\
\text { localized the affected ar- } \\
\text { eas. }\end{array}$ & $\begin{array}{l}\text { Although they used x- } \\
\text { ray images of most dis- } \\
\text { eases, they used only } 3 \\
\text { classes: COVID-19, } \\
\text { healthy, and disease }\end{array}$ \\
\hline Song et al [24] & $\mathrm{CT}^{\mathrm{b}}$ & 777 & 708 & N/A & 86 & $\begin{array}{l}\text { Proposed DRE-Net and } \\
\text { compared its perfor- } \\
\text { mance with VGG-16, } \\
\text { DenseNet, and ResNet }\end{array}$ & $\begin{array}{l}\text { Used only } 3 \text { classes: } \\
\text { COVID-19, bacterial } \\
\text { pneumonia, and healthy }\end{array}$ \\
\hline Zheng et al [25] & CT & 313 & 229 & N/A & 90 & $\begin{array}{l}\text { Proposed DeCoVNet for } \\
\text { classification }\end{array}$ & $\begin{array}{l}\text { Used only } 2 \text { classes: } \\
\text { COVID-19-positive } \\
\text { and COVID-19-nega- } \\
\text { tive }\end{array}$ \\
\hline Xu et al [26] & $\mathrm{X}$-ray & 219 & 175 & 224 & 86 & $\begin{array}{l}\text { Proposed ResNet-18 } \\
\text { based } \mathrm{CNN}^{\mathrm{c}} \text { network }\end{array}$ & $\begin{array}{l}\text { Used only } 3 \text { classes: } \\
\text { COVID-19, Influenza- } \\
\text { A viral pneumonia, and } \\
\text { normal }\end{array}$ \\
\hline Ozturk et al [27] & X-ray & 250 & 1000 & 500 & 92 & Proposed DarkCovidNet & $\begin{array}{l}\text { Used only } 3 \text { classes: } \\
\text { COVID- } 19 \text {, pneumonia, } \\
\text { and no findings }\end{array}$ \\
\hline $\begin{array}{l}\text { Ardakani et al } \\
\text { [28] }\end{array}$ & $\mathrm{CT}$ & 510 & N/A & 510 & 99 & $\begin{array}{l}\text { Used } 10 \mathrm{CNN} \text { networks } \\
\text { (ie, AlexNet and ResNet- } \\
101 \text { ) for classification of } \\
2 \text { classes }\end{array}$ & $\begin{array}{l}\text { Classified COVID-19 } \\
\text { class from } \\
\text { non-COVID-19 class }\end{array}$ \\
\hline Li et al [18] & CT & 1296 & 1325 & 1735 & 96 & $\begin{array}{l}\text { Proposed COV-Net for } \\
\text { classifying } 3 \text { classes }\end{array}$ & $\begin{array}{l}\text { Used only } 3 \text { classes: } \\
\text { COVID-19, communi- } \\
\text { ty-acquired pneumonia, } \\
\text { and non-pneumonia }\end{array}$ \\
\hline Abbas et al [15] & $\mathrm{X}$-ray & 105 & 80 & 11 & 95.12 & $\begin{array}{l}\text { Proposed DeTrac- } \\
\text { ResNet18 CNN that uses } \\
\text { Decompose, Transfer, } \\
\text { and Compose architec- } \\
\text { ture }\end{array}$ & $\begin{array}{l}\text { Used only } 3 \text { classes: } \\
\text { normal, COVID-19, } \\
\text { and SARS }\end{array}$ \\
\hline Chen et al [16] & CT & 51 & N/A & 55 & 95.24 & $\begin{array}{l}\text { Used UNet++ along with } \\
\text { Keras for segmentation } \\
\text { and COVID-19 detection }\end{array}$ & $\begin{array}{l}\text { Used only binary classi- } \\
\text { fication for COVID-19 } \\
\text { detection }\end{array}$ \\
\hline
\end{tabular}

${ }^{\mathrm{a}} \mathrm{N} / \mathrm{A}$ : not applicable.

${ }^{\mathrm{b}} \mathrm{CT}$ : computed tomography.

${ }^{\mathrm{c}} \mathrm{CNN}$ : convolutional neural network. 


\section{Methods}

\section{Dataset}

The first step involved preprocessing of the data, which includes segmentation of the lungs and the heart from the whole image, as an x-ray image contains many unnecessary details. To perform this segmentation task, we trained the UNet model on segmented CXR data obtained by the Japanese Society of Radiological Technology, which were downloaded from their official website [29], and their corresponding masks, which were downloaded from the SCR database [30]. This dataset contains 247 images. For classification purposes, data for
COVID-19 was collected from Cohen et al's COVID Chest $\mathrm{X}$-ray dataset [31]. This dataset contains $\mathrm{X}$-ray images of many other diseases. Furthermore, $\mathrm{x}$-ray images from the datasets were separated using the available metadata file. Data for the other 14 chest diseases were provided by the National Institute of Health (NIH) and can be downloaded from the NIH Chest $\mathrm{X}$-ray Dataset of 14 Common Thorax Disease Categories [32]. Data available on the NIH Clinical Center website contains 112,120 images, belonging to 15 classes, which include 14 disease classes and 1 normal class - all of which were extracted through the available metadata file. The number of images per class is presented in Table 2.

Table 2. Number of images per class in the National Institute of Health Chest X-ray Dataset of 14 Common Thorax Disease Categories [32].

\begin{tabular}{|c|c|c|}
\hline Model and class & Training set, $\mathrm{n}$ & Testing set, $\mathrm{n}$ \\
\hline \multicolumn{3}{|l|}{ Model 1} \\
\hline COVID-19 & 455 & 22 \\
\hline Normal & 1995 & 405 \\
\hline Other & 4600 & 730 \\
\hline \multicolumn{3}{|l|}{ Model 2} \\
\hline Atelectasis & 200 & 100 \\
\hline Cardiomegaly & 200 & 100 \\
\hline Consolidation & 200 & 100 \\
\hline Edema & 200 & 100 \\
\hline Effusion & 200 & 100 \\
\hline Emphysema & 200 & 100 \\
\hline Fibrosis & 200 & 100 \\
\hline Hernia & 150 & 100 \\
\hline Infiltration & 200 & 100 \\
\hline Mass & 200 & 100 \\
\hline Nodule & 200 & 100 \\
\hline Pleural thickening & 200 & 100 \\
\hline Pneumonia & 200 & 100 \\
\hline Pneumothorax & 200 & 100 \\
\hline
\end{tabular}

The data were randomly split into training and testing sets, as there were very few data related to COVID-19. The idea was to keep the training set as large as possible given the small number of images present. Image augmentation compensated for the lack of data. This was not an issue for model 2 images. For model 1, however, the lack of data can cause a change in testing accuracy. To compensate for this issue, we also applied data augmentation while testing.

\section{Data Preprocessing}

Every $\mathrm{x}$-ray image has a different contrast and illumination as they are taken under different lighting conditions. Therefore, in the first step of preprocessing, histogram equalization was applied. CXR images also contain unnecessary details, such as the collarbone, shoulders, neck, and torso region. To remove these unnecessary details, lungs and heart segmentation were applied. For this purpose, the UNet segmentation model was trained on images from the Japanese Society of Radiological Technology with their corresponding masks. The architecture of the UNet model is shown in Table 3 . The input image size fed to the network was $256 \times 256 \times 3$. The contraction part acts as an encoder that extracts the context from the image using downsampling through the max-pooling layer. The expansive path acts as a decoder that precisely localizes the segmentation part using transpose convolution layers. It is an end-to-end, fully connected network and does not contain any dense layers. It also restores the image through upsampling. 
Table 3. Architecture of UNet model.

\begin{tabular}{|c|c|c|c|}
\hline \multicolumn{2}{|c|}{ Path, layer, and type } & \multirow{2}{*}{$\begin{array}{l}\text { Kernel size } \\
N / A^{a}\end{array}$} & \multirow{2}{*}{$\frac{\text { Filters }}{\text { N/A }}$} \\
\hline 1 & Input Layer & & \\
\hline \multicolumn{4}{|c|}{ Contraction Path } \\
\hline 2 & Convolution & $3 \times 3$ & 16 \\
\hline 3 & Dropout $(0.1)$ & N/A & N/A \\
\hline 4 & Convolution & $3 \times 3$ & 16 \\
\hline 5 & MaxPooling & $2 \times 2$ & 1 \\
\hline 6 & Convolution & $3 \times 3$ & 32 \\
\hline 7 & Dropout $(0.1)$ & N/A & N/A \\
\hline 8 & Convolution & $3 \times 3$ & 32 \\
\hline 9 & MaxPooling & $2 \times 2$ & 1 \\
\hline 10 & Convolution & $3 \times 3$ & 64 \\
\hline 11 & Dropout $(0.2)$ & N/A & N/A \\
\hline 12 & Convolution & $3 \times 3$ & 64 \\
\hline 13 & MaxPooling & $2 \times 2$ & 1 \\
\hline 14 & Convolution & $3 \times 3$ & 128 \\
\hline 15 & Dropout $(0.2)$ & N/A & N/A \\
\hline 16 & Convolution & $3 \times 3$ & 128 \\
\hline 17 & MaxPooling & $2 \times 2$ & 1 \\
\hline 18 & Convolution & $3 \times 3$ & 256 \\
\hline 19 & Dropout $(0.3)$ & N/A & N/A \\
\hline 20 & Convolution & $3 \times 3$ & 256 \\
\hline \multicolumn{4}{|c|}{ Expansive Path } \\
\hline 21 & Transposed convolution & $2 \times 2$ & 128 \\
\hline 22 & Concatenate $(21,16)$ & N/A & N/A \\
\hline 23 & Convolution & $3 \times 3$ & 128 \\
\hline 24 & Dropout $(0.2)$ & N/A & N/A \\
\hline 25 & Convolution & $3 \times 3$ & 128 \\
\hline 26 & Transposed convolution & $2 \times 2$ & 64 \\
\hline 27 & Concatenate $(26,12)$ & N/A & N/A \\
\hline 28 & Convolution & $3 \times 3$ & 64 \\
\hline 29 & Dropout (0.2) & N/A & N/A \\
\hline 30 & Convolution & $3 \times 3$ & 64 \\
\hline 31 & Transposed convolution & $2 \times 2$ & 32 \\
\hline 32 & Concatenate $(31,8)$ & N/A & N/A \\
\hline 33 & Convolution & $3 \times 3$ & 32 \\
\hline 34 & Dropout $(0.1)$ & N/A & N/A \\
\hline 35 & Convolution & $3 \times 3$ & 32 \\
\hline 36 & Transposed convolution & $2 \times 2$ & 16 \\
\hline 37 & Concatenate $(36,4)$ & N/A & N/A \\
\hline 38 & Convolution & $3 \times 3$ & 16 \\
\hline 39 & Dropout $(0.1)$ & N/A & N/A \\
\hline 40 & Convolution & $3 \times 3$ & 16 \\
\hline
\end{tabular}




\begin{tabular}{clll}
\hline Path, layer, and type & & Kernel size & Filters \\
\hline 41 & Convolution (Sigmoid) & $1 \times 1$ & 1 \\
\hline
\end{tabular}

${ }^{\mathrm{a}} \mathrm{N} / \mathrm{A}$ : not applicable.

\section{Data Augmentation}

Before feeding the data to the network, image augmentation was applied to tackle the problem of fewer data, that is, in the case of COVID-19. For applying augmentation, the rotation range was set to $90^{\circ}$, the horizontal flip was set to true, and the vertical flip was also set to true. For each iteration, the image data generator used a different transformation of the original images. In the case of COVID-19, we had 445 input images and 20 iterations; therefore, the data generator used 8900 images for training in this case.

\section{Classification Models}

The main objective of this study was to classify COVID-19 $\mathrm{X}$-ray images from normal x-ray images and those of 14 other chest diseases. When a single model is trained for classifying 16 different classes, its accuracy tends to decrease, and in the case of COVID-19 detection, that is not acceptable. To solve this problem, a new pipeline was formed, which is illustrated in Figure 1. Two models were trained. The first model was trained to classify 3 classes: COVID-19, normal, and some other disease. The second model was trained to classify the 14 other chest and related diseases. Both models were trained separately. To automate the process, if the first model classified the $\mathrm{x}$-ray as "some other disease," then the second model was called to further classify the disease as one of 14 other chest diseases, using a simple "IF" condition. This architecture makes the classification process easy, as there are fewer features that need to be classified at the first stage.

Figure 1. Proposed pipeline of classification.

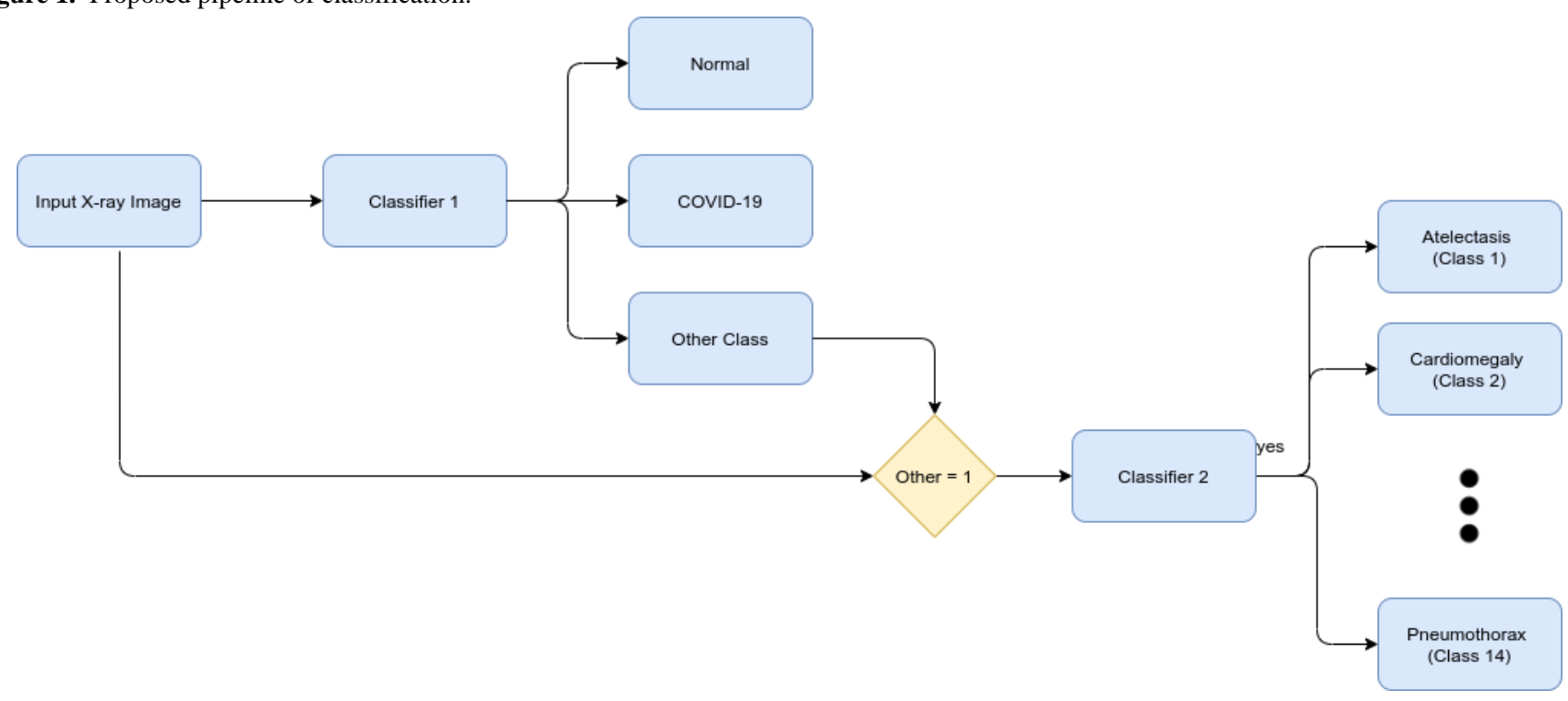

Classifier 1 only needs to learn how to distinguish COVID-19 and normal $\mathrm{x}$-ray images from those of all the other 14 chest diseases. The rule is simple: the fewer the classes, the fewer features there are to learn and distinguish, and the greater the accuracy. This is critical because the classification of COVID-19 is much more important than that of other diseases during the ongoing pandemic. Finally, the burden of classifying the other $14 \mathrm{x}$-ray diseases falls on classifier 2, which now has 14 classes to classify instead of 16 . Furthermore, the 2 most important classes have already been classified by classifier 1 . Moreover, to support the statement that accuracy indeed decreases when classifying into 16 classes, a third model was trained for classification into all 16 classes.

For classification purposes, the following 5 models were trained for both classifications:

NasNetLarge, Xception, InceptionV3, InceptionResNetV2, and ResNet50.
NasNetLarge was proposed by "Google Brain" in 2018 [33]. It has two types of architectures-CIFAR10 and ImageNet. CIFAR10 architecture has N number of normal cells and one reduction cell repeating after each other; in the end, it has the SoftMax function. ImageNet has two strides of convolutional layers with a $3 \times 3$ kernel size at the start, followed by two reduction cells; thereafter, it has the same architecture as CIFAR10.

Xception was proposed by Google in 2017 [34]. It consists of one entry flow, eight middle flow, and one exit flow. Entry flow consists of convolutional and max-pooling layers with ReLU as the activation function. The middle flow consists of only convolutional layers with the ReLU activation function. Exit flow consists of convolutional, max pooling, and global average pooling layers with the ReLU activation function; in the end, it has fully connected layers for classification.

InceptionV3 was proposed by Google in 2015 [35]. The basic architecture of the model is the same, as it consists of convolutional and pooling layers; in addition, it has three 
inception architectures as proposed previously [35]. Finally, at the end, it has the logistic and SoftMax function for classification into 1000 classes.

InceptionResNetV2 was proposed by Google in 2016 [36]. It has the proposed inception and reduction blocks at the start, and in the end, it has a pooling layer and dropout layer to prevent overfitting. It classifies using the SoftMax function.

ResNet50 was proposed by Microsoft in 2015 [37]. It takes residual learning as a building block and consists of convolutional layers with an average pooling layer at the end.
Models were taken from Keras library in Python, which were initialized with ImageNet weights. These models can classify 1000 classes, but we only needed to classify 3,14 , and 16 classes for classifier 1, classifier 2, and classifier 3, respectively. Therefore, these models were fine-tuned, and additional layers were added. Table 4 shows the fine-tuning layers added at the end of each pretrained model. The input image size given to the models was $331 \times 331 \times 3$.

Table 4. Fine-tuning layers for classifier 1, classifier 2, and classifier 3.

\begin{tabular}{lllllll}
\hline Type & Classifier 1 & & Classifier 2 & & \multicolumn{2}{c}{ Classifier 3 } \\
& Output & Kernel & Output & Kernel & Output & Kernel \\
\hline Average pooling & 2048 & $2 \times 2$ & 2048 & $2 \times 2$ & 2048 & N/A \\
Flatten & 8192 & N/A & 8192 & N/A & 8192 & 1024 \\
Dense & 1024 & N/A & 1024 & N/A & N/A \\
Dropout (0.5) & 1024 & N/A & 1024 & N/A & 1024 & N/A \\
Dense & 1024 & N/A & 1024 & N/A & 1024 & N/A \\
Dropout (0.5) & 1024 & N/A & 1024 & N/A & 1024 & N/A \\
Dense & 3 & N/A & 1024 & N/A & 16 & N/A \\
\hline
\end{tabular}

${ }^{\mathrm{a}} \mathrm{N} / \mathrm{A}$ : not applicable.

All the models explained in the Methods section were trained and tested on Google Colab with 12 GB of RAM and GPU (graphics processing unit) assigned by Google Colab.

\section{Results}

Initially, the UNet model was trained for segmentation of lungs and heart. After Training UNet, the model had a training loss

Figure 2. Sample segmented chest $\mathrm{x}$-ray images.

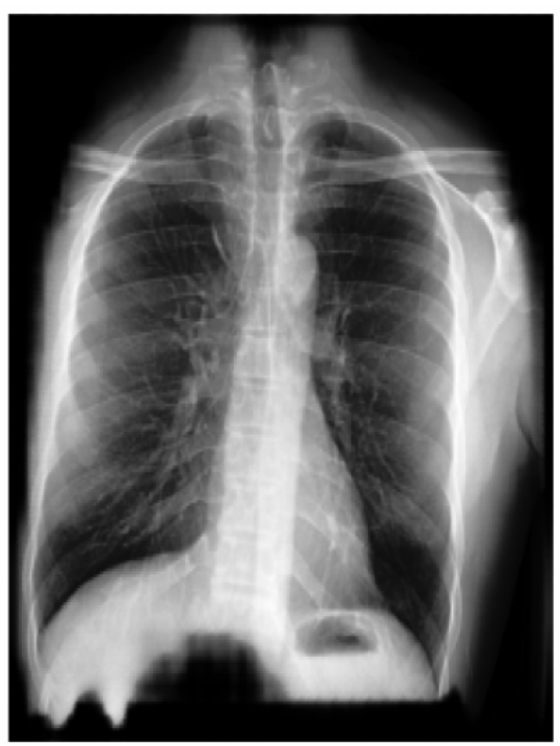

After image segmentation was completed and new training data were obtained, each training model was trained for 20 epochs with a batch size of 8 . The accuracy obtained from training is of $16.75 \%$, training accuracy of $87.13 \%$, validation loss of $12.27 \%$, and validation accuracy of $89.64 \%$.

Figure 2 shows some sample segmented CXR images. With image segmentation, we achieved up to $5 \%$ increase in the accuracy of our models.

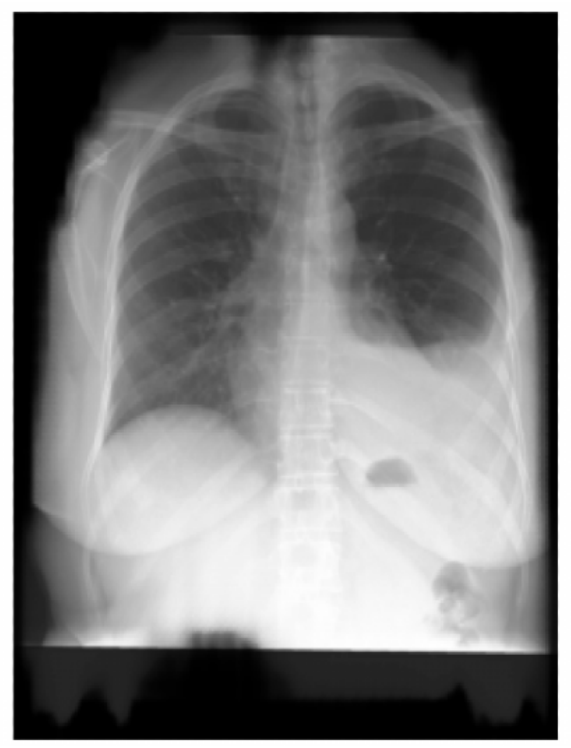

shown in Table 5. The table shows that the maximum accuracy for classifying the 3 classes, including COVID-19 was achieved by using ResNet50 followed by NasNetLarge. These two models 
yielded accuracy that competes with that of the available state-of-the-art models for COVID-19 prediction.

Classifier 2 did not show promising results in classifying the 14 other diseases. The main reasons for this were the large number of classes and the continued overfitting of the model. The maximum test accuracy achieved was $65.63 \%$ with ResNet50 followed by $61.47 \%$ with NasNetLarge.

As described, our proposed model pipeline helps to increase the accuracy of COVID-19 diagnosis when classifying 16 classes. Table 5 shows that when the 16 classes were combined and classified, the detection accuracy decreases. In all the cases except in the case of NASNetLarge and ResNet50 models, the test accuracy decreased when classifying 16 classes. Moreover, in the case of the NASNetLarge model, the increase in accuracy is not very notable. The maximum test accuracy was achieved with ResNet50, with an average of $71.905 \%$ over 10 -fold cross-validation.

Table 5. Average training, validation, and test accuracy achieved by different models through a 10-fold cross-validation.

\begin{tabular}{|c|c|c|c|c|c|c|}
\hline Model and classifier & Training accuracy $(\%)$ & Training loss $(\%)$ & $\begin{array}{l}\text { Validation accuracy } \\
(\%)\end{array}$ & Validation loss $(\%)$ & Test accuracy (\%) & Test loss (\%) \\
\hline \multicolumn{7}{|l|}{ NasNetLarge } \\
\hline $1 \mathrm{st}$ & 91.80 & 33.78 & 91.25 & 31.32 & 89.66 & 32.028 \\
\hline 2nd & 84.67 & 52.45 & 61.22 & 127.83 & 61.47 & 127.99 \\
\hline Combined & 79.72 & 68.36 & 63.68 & 123.42 & 63.58 & 121.39 \\
\hline \multicolumn{7}{|l|}{ Xception } \\
\hline $1 \mathrm{st}$ & 88.12 & 29.27 & 87.33 & 36.27 & 86.58 & 35.91 \\
\hline 2 nd & 90.70 & 48.73 & 61.88 & 133.04 & 61.08 & 133.92 \\
\hline Combined & 29.88 & 208.89 & 22.28 & 397.08 & 47.75 & 301.39 \\
\hline \multicolumn{7}{|l|}{ InceptionV3 } \\
\hline $1 \mathrm{st}$ & 65.87 & 69.25 & 63.43 & 57.38 & 63.19 & 5.233 \\
\hline 2 nd & 83.91 & 56.46 & 53.57 & 142.22 & 53.75 & 139.97 \\
\hline Combined & 65.52 & 110.33 & 38.31 & 176.24 & 38.83 & 174.97 \\
\hline \multicolumn{7}{|l|}{ InceptionResNetV2 } \\
\hline $1 \mathrm{st}$ & 65.10 & 80.32 & 62.46 & 76.35 & 63.19 & 75.79 \\
\hline 2nd & 83.37 & 81.30 & 54.08 & 197.66 & 53.75 & 197.07 \\
\hline Combined & 54.45 & 134.20 & 33.84 & 200.61 & 33.97 & 200.26 \\
\hline \multicolumn{7}{|l|}{ ResNet50 } \\
\hline $1 \mathrm{st}$ & 96.32 & 9.84 & 94.16 & 23.09 & 92.52 & 20.32 \\
\hline $2 \mathrm{nd}$ & 87.83 & 35.85 & 67.55 & 105.63 & 65.63 & 108.24 \\
\hline Combined & 88.92 & 26.16 & 73.14 & 87.05 & 71.91 & 88.95 \\
\hline
\end{tabular}

The results obtained by our proposed approaches compete with that of state-of-the-art methods (shown in Table 1). Graphs illustrating the training and validation accuracy and loss for classifiers 1, 2, and 3 are shown in Figures 3, 4, and 5, respectively. To further evaluate the results, the AUC (area under the curve), sensitivity, and specificity results for all the networks were studied (Table 6). We found that ResNet50 achieved the maximum AUC, sensitivity, and specificity scores compared to any other model. 
Figure 3. Graphs illustrating training and validation accuracy (left) and loss (right) over epochs for different models of classifier 1.
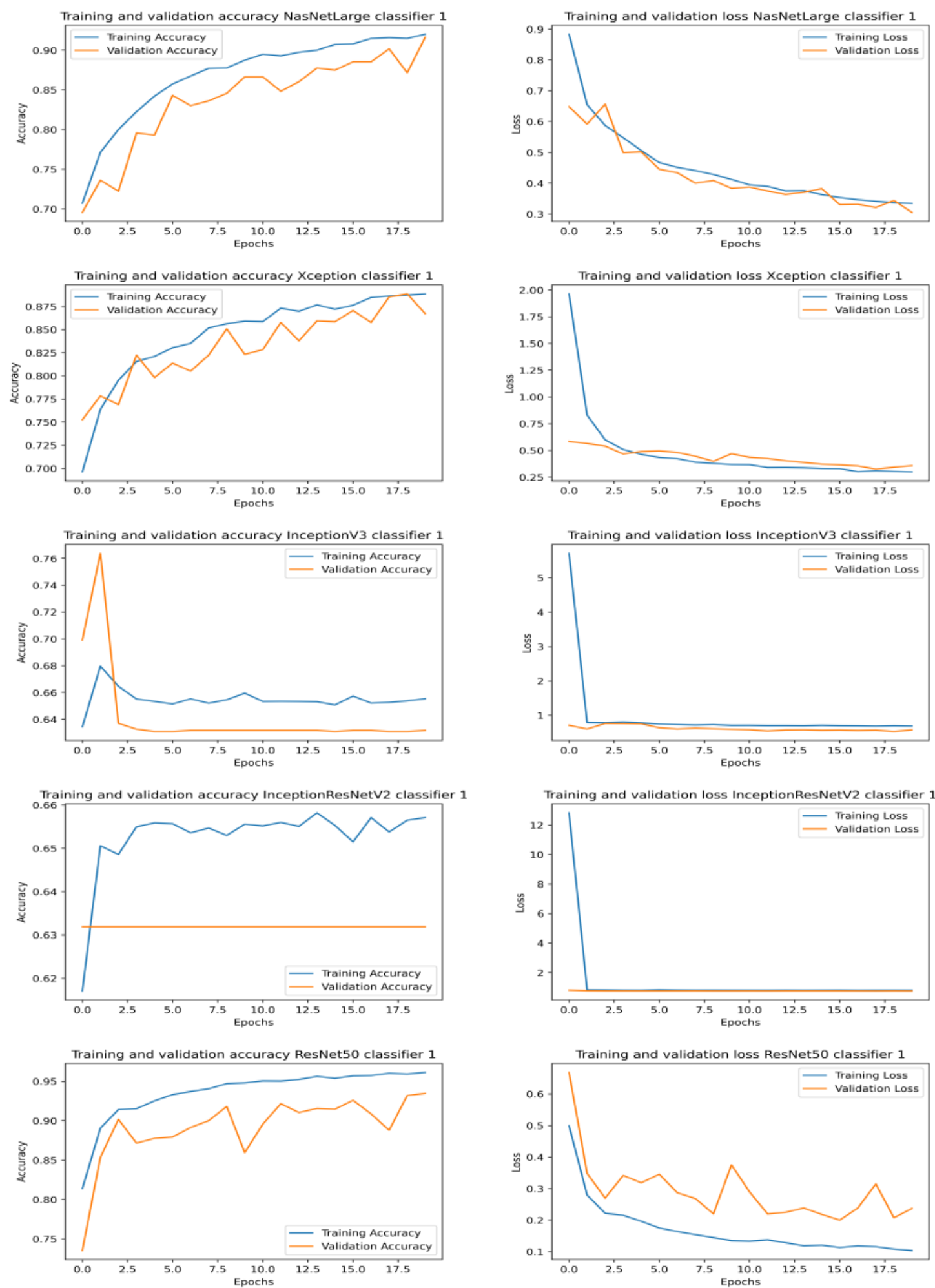
Figure 4. Graphs illustrating training and validation accuracy (left) and loss (right) over epochs for different models of classifier 2.
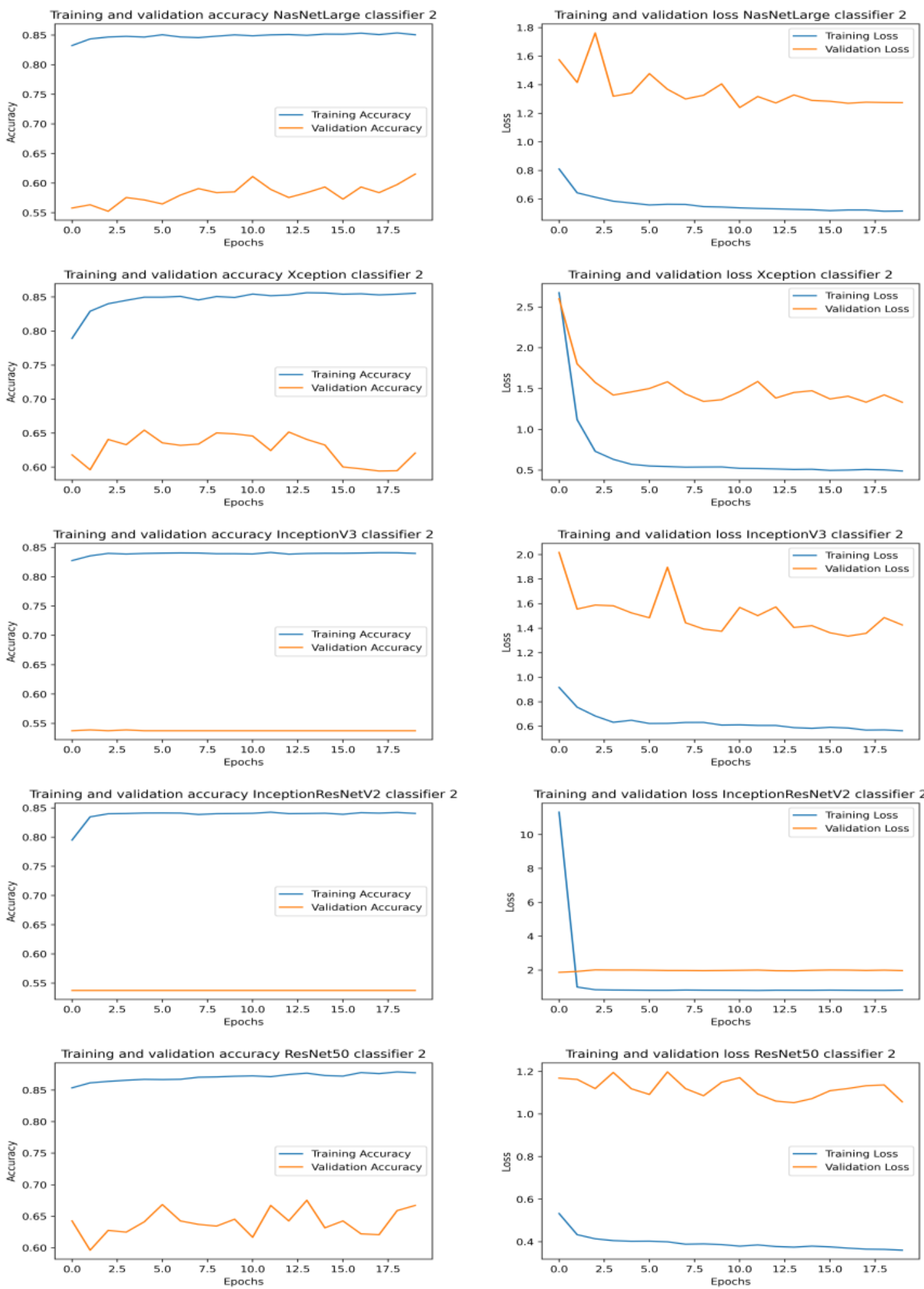
Figure 5. Graphs illustrating training and validation accuracy (left) and loss (right) over epochs for different models of classifier 3.
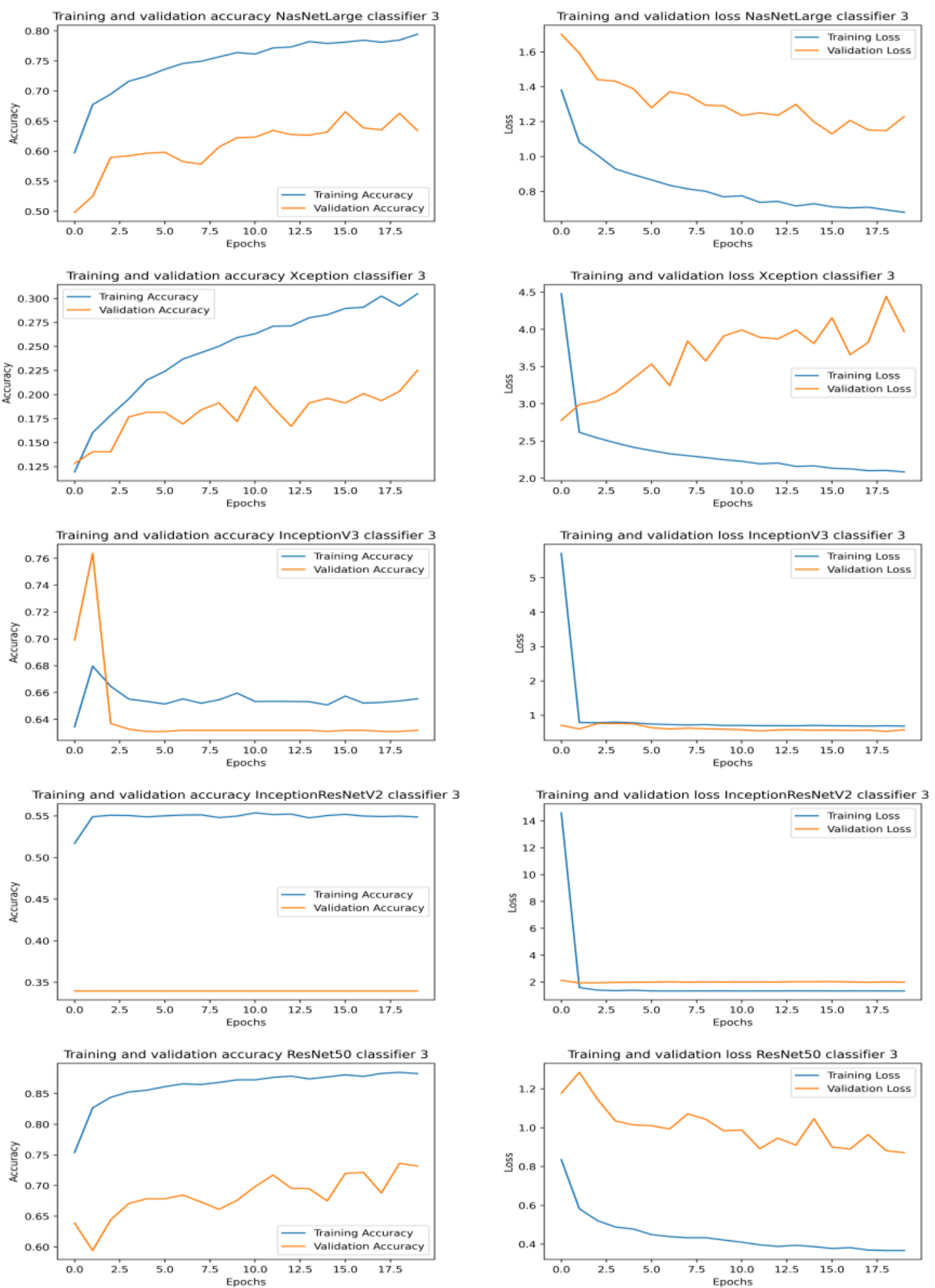
Table 6. Average training, validation, and test accuracy achieved by different models through the 10 -fold cross-validation.

\begin{tabular}{|c|c|c|c|}
\hline Model and classifier & $\operatorname{AUC}^{\mathrm{a}}(\%)$ & Sensitivity (\%) & Specificity (\%) \\
\hline \multicolumn{4}{|l|}{ NasNetLarge } \\
\hline 1 st & 97.61 & 90.73 & 93.42 \\
\hline 2nd & 82.15 & 75.33 & 81.1 \\
\hline Combined & 93.88 & 90.15 & 93.85 \\
\hline \multicolumn{4}{|l|}{ Xception } \\
\hline 1 st & 95.9 & 88.64 & 91.78 \\
\hline 2nd & 92.25 & 87.38 & 81.12 \\
\hline Combined & 83.19 & 76.09 & 80.64 \\
\hline \multicolumn{4}{|l|}{ InceptionV3 } \\
\hline 1 st & 89.28 & 83.2 & 85.51 \\
\hline 2nd & 91.69 & 79.53 & 81.25 \\
\hline Combined & 89.85 & 83.29 & 93.7 \\
\hline \multicolumn{4}{|l|}{ InceptionResNetV2 } \\
\hline 1 st & 80.65 & 74.88 & 77.92 \\
\hline 2 nd & 85.41 & 79.21 & 81.25 \\
\hline Combined & 85.44 & 82.69 & 93.75 \\
\hline \multicolumn{4}{|l|}{ ResNet50 } \\
\hline 1 st & 98.73 & 93.14 & 95.22 \\
\hline 2nd & 94.6 & 85.64 & 81.03 \\
\hline Combined & 96.9 & 93.4 & 93.72 \\
\hline
\end{tabular}

${ }^{\mathrm{a}}$ Area under the curve.

\section{Discussion}

\section{Principal Findings}

In this study, we classified normal cases, COVID-19 cases, and 14 other chest diseases based on CXR images. We proposed a novel, multiclass method for this purpose and used models that were pretrained on ImageNet dataset to save training time and resources. Our multilevel approach resulted in an increase in the classification accuracy. We found that ResNet50 was the best model for classification, yielding the highest accuracy.

\section{Future Suggestions}

This study tried to cover most aspects of detection of chest diseases, but there is still work to be done. Most importantly, there is a need for more data for patients with COVID-19, which could help improve the accuracy of the model. At present, there is a significant difference in the number of images per class for the first level of classification.

This model can help in the first level of classification to determine whether the person has COVID-19 or some other chest disease, as X-rays are easier and less expensive than other forms of radiographic imaging and can help determine the severity of the disease. Although disease severity was not within the scope of this study, future work in detecting the severity of the disease can also be an important improvement in the already-existing model. In addition, techniques such as the
Grad-Cam algorithm can be used to visualize the features in radiographic images affecting the algorithm and to determine disease severity. This algorithm will highlight which features help the algorithm with the classification and which features likely mislead the algorithm. This algorithm might also be the key to investigating the low accuracy of the level-2 classifier and can help improve its accuracy.

\section{Conclusions}

Deep learning has played a major role in medical image analysis and feature extraction, which are applied to the detection of a wide range of chest diseases. $\mathrm{CNN}$ architectures are popular for their ability to learn mid- and high-level image representations and to make predictions. Detecting the presence, or absence, of COVID-19 in a patient is insufficient without addressing other chest diseases. However, a deep learning system that is trained to classify a large number of classes - 16 in our case- has less accuracy. This work aimed to deal effectively with this new pipeline to help with a first-level differential diagnosis of COVID-19 from other chest diseases. Subsequently, we applied further enhancement to detect other chest diseases in order to tackle multi-class chest classification in the detection of anomalies on X-ray images. This approach yielded satisfactory results.

Thus, we showed how our proposed models use state-of-the-art deep neural networks to classify 16 cardiothoracic diseases by training the models based on $\mathrm{x}$-ray images in the database. Image 
segmentation was applied to remove unnecessary details, and both classifiers were independently trained on segmented data. However, our model can classify not only COVID-19 but also
14 other chest diseases, as well as normal x-ray images, with satisfactory accuracy as compared with previous studies.

\section{Acknowledgments}

We would like to thank the Deanship of Scientific Research, Qassim University, for funding the publication of this project.

\section{Conflicts of Interest}

None declared.

\section{References}

1. Coronavirus disease 2019 (COVID-19) Situation Report - 94. World Health Organization. 2019. URL: https://www.who.int/ docs/default-source/coronaviruse/situation-reports/20200423-sitrep-94-covid-19.pdf [accessed 2021-02-04]

2. Yang X, Yu Y, Xu J, Shu H, Xia J, Liu H, et al. Clinical course and outcomes of critically ill patients with SARS-CoV-2 pneumonia in Wuhan, China: a single-centered, retrospective, observational study. The Lancet Respiratory Medicine 2020 May;8(5):475-481. [doi: 10.1016/s2213-2600(20)30079-5]

3. Albahli S. A deep neural network to distinguish COVID-19 from other chest diseases using X-ray images. Curr Med Imaging. Epub ahead of print posted online on June 4, 2020 2020. [doi: 10.2174/1573405616666200604163954] [Medline: 32496988]

4. Haglin JM, Jimenez G, Eltorai AEM. Artificial neural networks in medicine. Health Technol 2018 Jul 27;9(1):1-6. [doi: 10.1007/s12553-018-0244-4]

5. Choe J, Lee SM, Do K, Lee G, Lee J, Lee SM, et al. Deep learning-based image conversion of CT reconstruction kernels improves radiomics reproducibility for pulmonary nodules or masses. Radiology 2019 Aug;292(2):365-373. [doi: 10.1148/radiol.2019181960] [Medline: 31210613 ]

6. Sivasamy J, Subashini T. Classification and predictions of lung diseases from chest X-rays using MobileNet. The International Journal of Analytical and Experimental Modal Analysis 2020 Mar;12(3):665-672 [FREE Full text]

7. Wang Y, Zhang Y, Xuan W, Kao E, Cao P, Tian B, et al. Fully automatic segmentation of 4D MRI for cardiac functional measurements. Med Phys 2019 Jan;46(1):180-189 [FREE Full text] [doi: 10.1002/mp.13245] [Medline: $\underline{30352129}$ ]

8. Rajpurkar P, Irvin J, Zhu K, Yang B, Mehta H, Duan T, et al. CheXNet: Radiologist-level pneumonia detection on chest X-rays with deep learning. arXiv. Preprint posted online on December 25, 2017 [FREE Full text]

9. Salehinejad H, Valaee S, Dowdell T, Colak E, Barfett J. Generalization of deep neural networks for chest pathology classification in X-rays using generative adversarial networks. : IEEE; 2018 Presented at: 2018 IEEE International Conference on Acoustics, Speech and Signal Processing (ICASSP); April 15-20, 2018; Calgary, AB, Canada p. 990-994. [doi: 10.1109/icassp.2018.8461430]

10. Chandra TB, Verma K. Pneumonia detection on chest X-ray using machine learning paradigm. In: Chaudhuri B, Nakagawa M, Khanna P, Kumar S, editors. roceedings of 3rd International Conference on Computer Vision and Image Processing. Advances in Intelligent Systems and Computing, vol 1022. Singapore: Springer; 2019:21-33.

11. Wang X, Peng Y, Lu L, Lu Z, Bagheri M, Summers RM. Hospital-scale chest X-ray database and benchmarks on weakly-supervised classification and localization of common thorax diseases. : IEEE; 2017 Presented at: Proceedings of the IEEE conference on computer vision and pattern recognition; July 21-26, 2017; Honolulu, HI p. 2097-2106. [doi: 10.1109/cvpr.2017.369]

12. Smit A, Jain S, Rajpurkar P, Pareek A, Ng AY, Lungren MP. Combining automatic labelers and expert annotations for accurate radiology report labeling using BERT. In: ACL Anthology.: Association for Computational Linguistics; 2020 Presented at: Proceedings of the 2020 Conference on Empirical Methods in Natural Language Processing (EMNLP); November 2020; Online p. 1500-1519. [doi: 10.18653/v1/2020.emnlp-main.117]

13. Ho TK, Gwak J. Multiple feature integration for classification of thoracic disease in chest radiography. Applied Sciences 2019 Oct 02;9(19):4130. [doi: 10.3390/app9194130]

14. Apostolopoulos ID, Mpesiana TA. Covid-19: automatic detection from X-ray images utilizing transfer learning with convolutional neural networks. Phys Eng Sci Med 2020 Jun;43(2):635-640 [FREE Full text] [doi: 10.1007/s13246-020-00865-4] [Medline: 32524445]

15. Abbas A, Abdelsamea M, Gaber MM. Classification of COVID-19 in chest X-ray images using DeTraC deep convolutional neural network. Appl Intell 2020 Sep 05;51(2):854-864. [doi: 10.1007/s10489-020-01829-7]

16. Chen J, Wu L, Zhang J, Zhang L, Gong D, Zhao Y, et al. Deep learning-based model for detecting 2019 novel coronavirus pneumonia on high-resolution computed tomography: a prospective study. medRxiv. Preprint posted online on March 01, 2020 [FREE Full text] [doi: 10.1101/2020.02.25.20021568]

17. Narin A, Kaya C, Pamuk Z. Automatic detection of Coronavirus disease (covid-19) using X-ray images and deep convolutional neural networks. arXiv. Preprint posted online on March 24, 2020 [FREE Full text] 
18. Li L, Qin L, Xu Z, Yin Y, Wang X, Kong B, et al. Using artificial intelligence to detect COVID-19 and community-acquired pneumonia based on pulmonary CT: evaluation of the diagnostic accuracy. Radiology 2020 Aug;296(2):E65-E71 [FREE Full text] [doi: 10.1148/radiol.2020200905] [Medline: $\underline{\text { 32191588] }}$

19. Sethy PK, Behera SK. Detection of Coronavirus disease (covid-19) based on deep features. Preprints. Preprint posted online on March 18, 2020 [FREE Full text] [doi: 10.20944/preprints202003.0300.v1]

20. Hemdan ED, Shouman MA, Karar ME. Covidx-net: A framework of deep learning classifiers to diagnose covid-19 in X-ray images. arXiv. Preprint posted online on March 24, 2020 [FREE Full text]

21. Wang S, Kang B, Ma J, Zeng X, Xiao M, Guo J, et al. A deep learning algorithm using ct images to screen for Coronavirus disease (covid-19). medRxiv. Preprint posted online on March 11, 2020 [FREE Full text]

22. Brunese L, Mercaldo F, Reginelli A, Santone A. Explainable deep learning for pulmonary disease and coronavirus COVID-19 detection from x-rays. Comput Methods Programs Biomed 2020 Nov;196:105608 [FREE Full text] [doi:

10.1016/j.cmpb.2020.105608] [Medline: $\underline{32599338]}$

23. Wang L, Lin ZQ, Wong A. COVID-Net: a tailored deep convolutional neural network design for detection of COVID-19 cases from chest X-ray images. Sci Rep 2020 Nov 11;10(1):19549 [FREE Full text] [doi: 10.1038/s41598-020-76550-z] [Medline: $\underline{33177550]}$

24. Song Y, Zheng S, Li L, Zhang X, Ziwang Huang Z, Chen H, et al. Deep learning enables accurate diagnosis of novel coronavirus (COVID-19) with CT images. medRxiv. Preprint posted online on February 25, 2020 [FREE Full text] [doi: $10.1101 / 2020.02 .23 .20026930]$

25. Zheng C, Deng X, Fu Q, Zhou Q, Feng J, Ma H, et al. Deep learning-based detection for COVID-19 from chest CT using weak label. medRxiv. Preprint posted online on March 26, 2020 [FREE Full text] [doi: 10.1101/2020.03.12.20027185]

26. Xu X, Jiang C, Ma C. Deep learning system to screen coronavirus disease 2019 pneumonia. arXiv. Preprint posted online on February 21, 2020 [FREE Full text]

27. Ozturk T, Talo M, Yildirim EA, Baloglu UB, Yildirim O, Rajendra Acharya U. Automated detection of COVID-19 cases using deep neural networks with X-ray images. Comput Biol Med 2020 Jun;121:103792 [FREE Full text] [doi: 10.1016/j.compbiomed.2020.103792] [Medline: 32568675]

28. Ardakani AA, Kanafi AR, Acharya UR, Khadem N, Mohammadi A. Application of deep learning technique to manage COVID-19 in routine clinical practice using CT images: Results of 10 convolutional neural networks. Comput Biol Med 2020 Jun;121:103795 [FREE Full text] [doi: 10.1016/j.compbiomed.2020.103795] [Medline: 32568676]

29. JSRT chest X-ray dataset. Japanese Society of Radiological Technology. URL: http://db.jsrt.or.jp/eng-04.php [accessed 2021-02-04]

30. SCR Segmentation Maps for JSRT Chest X-ray Dataset. SCR Segmentation Maps for JSRT Chest X-ray Dataset. SCR Database: Download. URL: http://www.isi.uu.nl/Research/Databases/SCR/download.php [accessed 2021-02-04]

31. Cohen JP, Morrison P, Dao L, Roth K, Duong TQ, Ghassemi M. COVID-19 image data collection: prospective predictions are the future. arXiv. Preprint posted online on June 22, 2020. https://github.com/ieee8023/covid-chestxray-dataset [FREE Full text]

32. NIH Chest X-ray Dataset of 14 Common Thorax Disease Categories. National Institutes of Health - Clinical Center. URL: https://academictorrents.com/details/557481faacd824c83fbf57dcf7b6da9383b3235a [accessed 2021-02-04]

33. Zoph B, Vasudevan V, Shlens J, Le QV. Learning Transferable Architectures for Scalable Image Recognition. : IEEE; 2018 Presented at: 2018 IEEE/CVF Conference on Computer Vision and Pattern Recognition; June 18-23, 2018; Salt Lake City, UT p. 8697-8710 URL: https://ieeexplore.ieee.org/document/8579005 [doi: 10.1109/cvpr.2018.00907]

34. Chollet F. Xception: Deep Learning with Depthwise Separable Convolutions. : IEEE; 2017 Presented at: 2017 IEEE Conference on Computer Vision and Pattern Recognition (CVPR); July 21-26, 2017; Honolulu, HI p. 1800-1807 URL: https://ieeexplore.ieee.org/document/8099678 [doi: 10.1109/cvpr.2017.195]

35. Szegedy C, Vanhoucke V, Ioffe S, Shlens J, Wojna Z. Rethinking the Inception Architecture for Computer Vision. : IEEE; 2016 Presented at: 2016 IEEE Conference on Computer Vision and Pattern Recognition (CVPR); June 27-30, 2016; Las Vegas, NV p. 2818-2826 URL: https://ieeexplore.ieee.org/document/7780677 [doi: 10.1109/CVPR.2016.308]

36. Szegedy C, Ioffe S, Vanhoucke V, Alemi AA. Inception-v4, inception-ResNet and the impact of residual connections on learning. : AAAI Press; 2017 Presented at: AAAI'17: Proceedings of the Thirty-First AAAI Conference on Artificial Intelligence; February 2017; San Francisco, CA p. 4278-4284 URL: https://dl.acm.org/doi/10.5555/3298023.3298188

37. He K, Zhang X, Ren S, Sun J. Deep Residual Learning for Image Recognition. : IEEE; 2016 Presented at: 2016 IEEE Conference on Computer Vision and Pattern Recognition (CVPR); June 27-30, 2016; Las Vega, NV p. 770-778 URL: https://ieeexplore.ieee.org/document/7780459 [doi: 10.1109/cvpr.2016.90]

\section{Abbreviations}

AUC: area under the curve

CNN: convoluted neural network

CT: computed tomography

CXR: chest X-ray 
DCGAN: Deep Convolutional Generative Adversarial Network

NIH: National Institute of Health

SARS: severe acute respiratory syndrome

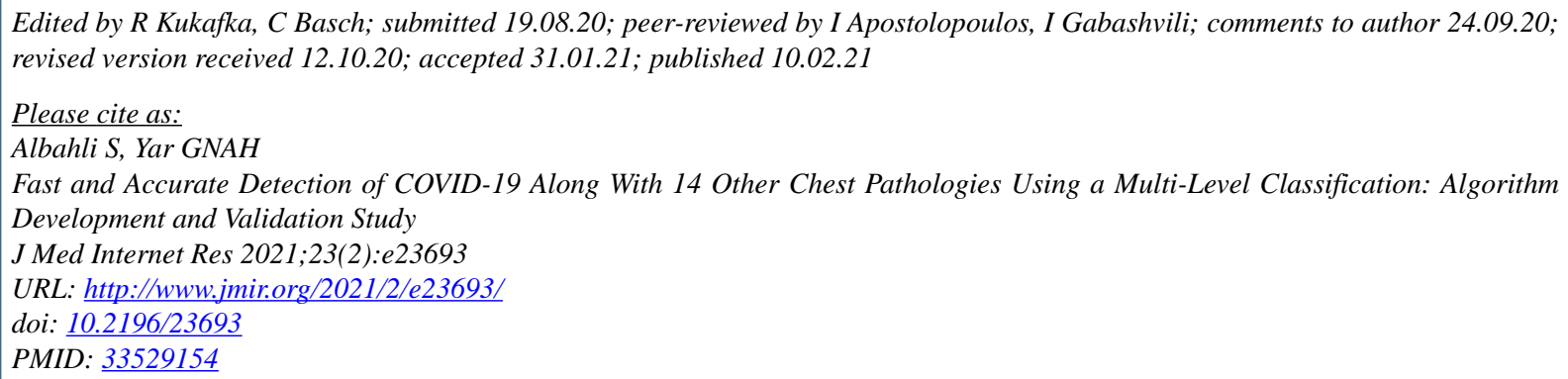

CSaleh Albahli, Ghulam Nabi Ahmad Hassan Yar. Originally published in the Journal of Medical Internet Research (http://www.jmir.org), 10.02.2021. This is an open-access article distributed under the terms of the Creative Commons Attribution License (https://creativecommons.org/licenses/by/4.0/), which permits unrestricted use, distribution, and reproduction in any medium, provided the original work, first published in the Journal of Medical Internet Research, is properly cited. The complete bibliographic information, a link to the original publication on http://www.jmir.org/, as well as this copyright and license information must be included. 